\title{
EFECTO DE LA ESBELTEZ EN LA RESISTENCIA DE MUROS DE MAMPOSTERÍA CONFINADA
}

\author{
Juan José Pérez Gavilán ${ }^{(1)}$, Leonardo Flores ${ }^{(2)}$ y Sergio Alcocer ${ }^{(3)}$
}

\begin{abstract}
RESUMEN
Se presentan los resultados de un estudio experimental consistente en el ensayo de siete muros de mampostería confinada de barro a escala natural con relaciones de aspecto $(H / L)$ que van desde 0.3 hasta 2.2. Se investigó el posible cambio de la rigidez lateral, antes del primer agrietamiento, con la carga vertical, y la degradación de la rigidez y resistencia laterales de los muros ante carga lateral cíclica. Los resultados muestran que la carga vertical y la relación de aspecto no tuvieron un efecto significativo en la rigidez lateral relativa a la teórica. Asimismo, que el comportamiento inelástico de los muros, caracterizado por medio de la degradación de la rigidez normalizada contra la demanda de ductilidad, puede estimarse, con buena precisión, con una función bilineal para valores de demanda de ductilidad hasta de 4.5. Se observó un incremento sustancial de la resistencia al agrietamiento normalizada con respecto a la resistencia nominal, con la reducción de la relación de aspecto. Se dedujo una expresión para estimar la resistencia al agrietamiento en función de la relación de aspecto, que coincide razonablemente con los resultados experimentales. Se propone una nueva expresión para el cálculo de la fuerza cortante de agrietamiento para diseño.
\end{abstract}

Palabras clave: mampostería confinada; esbeltez; resistencia a corte; relación de aspecto

\begin{abstract}
Results from an experimental program of seven full-scale confined masonry walls with height-tolength aspect ratios $(H / L)$ from 0.3 up to 2.2 are summarized. The potential change of lateral stiffness, before the first inclined cracking, with vertical load was investigated. Lateral stiffness and strength degradation of walls with cyclic lateral loads was studied. Results showed that neither the level of axial stress nor the aspect ratio had a significant effect on lateral stiffness. Also, the inelastic behavior of the walls, characterized by normalized stiffness degradation with ductility demand, can be estimated with good accuracy with a bilinear function for a ductility demand up to 4.5. A substantial increase in shear cracking strength, normalized relative to the nominal strength, with a reduction of aspect ratio was observed. An expression to estimate the normalized cracking shear strength, as a function of aspect ratio, was developed. Results from this expression are in good agreement with the experimental results. A new expression for cracking shear strength is proposed for design.
\end{abstract}

Keywords: confined masonry; slenderness; shear strength; aspect ratio

Artículo recibido el 2 de diciembre de 2012 y aprobado para su publicación el 24 de abril de 2013. Se aceptarán comentarios y/o discusiones hasta cinco meses después de su publicación.

(1) Mecánica aplicada, Instituto de Ingeniería de la UNAM, CU, CP 04510, jipge@pumas.iingen.unam.mx

(2) CENAPRED, Av. Delfín Madrigal No 665, Col Pedregal Sto. Domingo, CP 04360 México D. F., lfc@ cenapred.unam.mx

(3) Universidad Nacional Autónoma de México, CU, salcocerm@ii.unam.mx 


\section{INTRODUCCIÓN}

La mampostería confinada consiste en muros de mampostería rodeados por pequeñas columnas de concreto en el sentido vertical, conocidas en México como castillos y pequeñas vigas (dalas) en el sentido horizontal. Los castillos se cimbran y cuelan después de que el muro ha sido construido. Normalmente son externos al muro, o bien, pueden estar embebidos dentro de una pieza hueca, donde se coloca el acero longitudinal de refuerzo, que después se rellena de concreto. El sistema con castillos externos es ampliamente usado en México, Centro y Sudamérica, Medio Oriente y Europa del Este, mientras que el segundo sistema es menos común (Riahi et al., 2009). En la literatura, ambos sistemas son referidos como mampostería confinada (MC), aunque tienen marcadas diferencias en su comportamiento. En particular, el modo de falla por flexión, en el que interviene el aplastamiento del extremo del muro, es rara vez observado en el caso de castillos externos. Se ha demostrado mediante extensos trabajos experimentales (Alcocer y Meli, 1995; Tomazevic y Klemenc, 1997; Aguilar et al., 1996; Yoshimura et al., 1996; Meli 1973) que la mampostería confinada tiene una resistencia adecuada a cortante y flexión, y una capacidad razonable a la distorsión lateral que evita un comportamiento frágil. Las pruebas indican que la capacidad de deformación de los muros depende de muchas variables, entre ellas, la relación de aspecto (altura a longitud $H / L$ ), la relación de cortante a momento, las cuantías de refuerzo vertical y horizontal en el muro, el nivel de carga axial, el tipo de pieza y de si los huecos de las piezas están rellenos de mortero o no.

Hidalgo et al. $(1978,1979)$ y Chen et al. (1978) probaron 80 muros a escala natural de mampostería reforzada utilizando como principales variables el tipo de mampostería, la carga vertical, la cuantía de refuerzo, tanto horizontal como vertical, y la relación de aspecto. Los muros se ensayaron en tres series, cada una con distinta relación de aspecto, $H / L=0.5,1$ y 2, respectivamente. En estas pruebas se observó que la resistencia al agrietamiento y la resistencia máxima coincidían en muros con relación de aspecto 2 y 1 , mientras que para muros con $H / L=0.5$ se tenía una reserva de resistencia después del agrietamiento, mayor para el caso de mampostería de barro en comparación con la de concreto. Matsumura (1998), condujo seis series de pruebas en mampostería reforzada. En cada serie, todas las variables se mantuvieron constantes cambiando solo la relación de aspecto. La relación de aspecto se varió entre 1.0 y 0.4 , ensayando un total de 18 especímenes. Se evaluó la cuantía de refuerzo horizontal, el nivel de carga axial, el llenado de concreto de todos los huecos de las piezas o solo algunos de ellos y el tipo de pieza. El autor encontró una degradación hiperbólica de la resistencia cortante normalizada con la relación de aspecto. La normalización se obtuvo dividiendo la resistencia medida por el producto del área transversal del muro y la raíz cuadrada de la resistencia a compresión de la mampostería. Matsumura (1998) no dio una explicación teórica de las diferencias observadas en la resistencia a corte; se limitó a hacer un análisis de regresión para el desarrollo de las ecuaciones de comportamiento. Álvarez (1996) ha argumentado que la relación de aspecto debe tener un impacto en la resistencia debido a cambios en la distribución de esfuerzos en comparación con un muro cuadrado $(H / L=1)$. Para sustentar esa idea, Álvarez presentó evidencia experimental de varios autores que mostraba que la resistencia a corte crece al disminuir la relación de aspecto. Voon e Ingham (2006) llegaron a conclusiones similares, basados en el ensayo de tres muros $(H / L=0.6,1,2)$ de una serie de diez. Recientemente, Riahi et al. (2009) analizaron una extensa base de datos de muros, con el objetivo de desarrollar un modelo para la mampostería confinada. Con base en un análisis de regresión, encontraron que el tipo de pieza, el refuerzo transversal en los castillos y la relación de aspecto eran variables poco significativas por lo que fueron excluidas de los modelos de cortante al agrietamiento y a la falla. Sin embargo, en ese estudio se observaron variaciones moderadas en la resistencia al corte debido a que estuvo limitado a muros con un solo tablero confinado con castillos y relaciones de aspecto en el intervalo reducido de 0.7 a 1.2. Este hecho, y la gran dispersión de los datos que es inevitable en ese tipo de estudios, en el que los resultados provienen de muy diversas condiciones 
experimentales, hicieron difícil la obtención de una variación consistente de la resistencia al corte con la relación de aspecto.
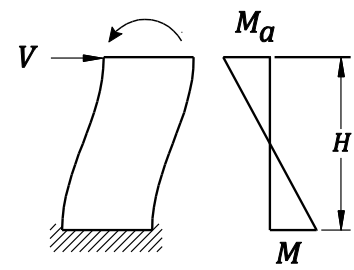

$=$
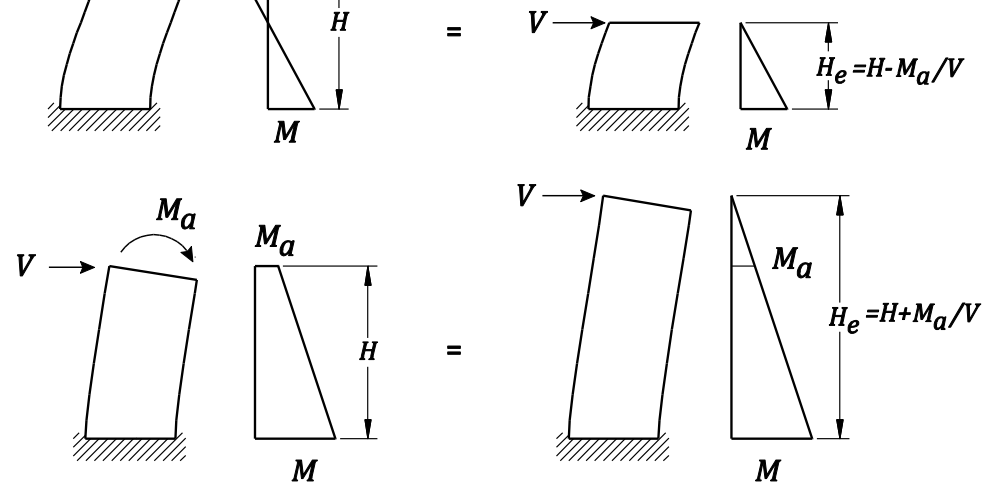

a)

b)

Figura 1. Altura efectiva, a) condiciones del muro consideradas usando b) una relación de aspecto efectiva $H_{e} / L=M / V L$, en vez de la relación de aspecto real $H / L$

Tabla 1. Fórmulas de resistencia a corte que toman en cuenta la relación de aspecto

\begin{tabular}{lcc}
\hline Código & Resistencia nominal a cortante & Requisitos \\
\hline $\begin{array}{c}\text { UBC SD } \\
\text { (1997) }\end{array}$ & $V_{n}=\left(\frac{7}{3}-\frac{4}{3} \frac{M}{V d}\right) \frac{6}{5} A \sqrt{f_{m}^{\prime}}+A \rho_{n} f_{y}<V_{n m}$ & $\frac{1}{4} \leq \frac{M}{V d} \leq 1$ \\
$\begin{array}{c}\text { MSJC SD } \\
(2002)\end{array}$ & $V_{n}=\left(\frac{16}{9}-\frac{7}{9} \frac{M}{V L}\right) \frac{9}{4} A \sqrt{f_{m}^{\prime}}+0.25 P+0.5\left(\frac{A_{v}}{s}\right) f_{y} L<V_{n m}$ & $\frac{1}{4} \leq \frac{M}{V L} \leq 1$ \\
CSA & $V_{n}=\left(2-\frac{M}{V d}\right) \frac{1}{6} A_{e} \sqrt{f_{m}^{\prime}}+0.25 P+0.6\left(\frac{A_{v}}{s}\right) f_{y} d<V_{n m}$ & Huecos colados \\
$(2004)$ & $V_{n}=\left(\frac{16}{9}-\frac{7}{9} \frac{H_{e}}{L}\right) \frac{9}{10} v_{b}+0.9 \frac{P}{A_{e}} \tan \alpha+c_{3}\left(\frac{A_{v}}{s}\right) f_{y}$ & Sin refuerzo vertical \\
NZS & $\frac{1}{4} \leq \frac{M}{V L} \leq 1$ \\
\hline
\end{tabular}

Nota: Las fórmulas corresponden a mampostería reforzada 


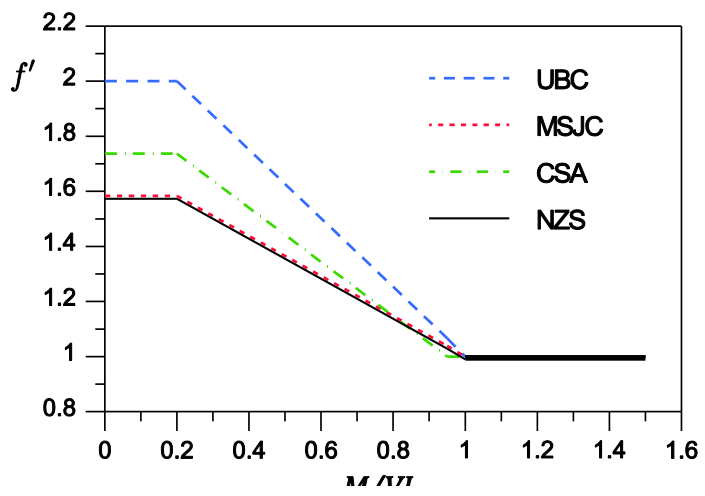

Figura 2. Variación de la resistencia básica de la mampostería con la relación de aspecto

Varios códigos incluyen en la fórmula de diseño por corte un factor que depende de la relación de aspecto, de modo de tomar en cuenta el crecimiento de la resistencia con la reducción de $H / L$, (Davis (2008) y Riahi et al. (2009). En la tabla 1 se muestran las fórmulas de resistencia a cortante de varios códigos. Las fórmulas están escritas en forma ligeramente distinta a la original, para facilitar su comparación.

En las fórmulas de resistencia que se muestran en la tabla 1, se utiliza el parámetro $M / V d$, o alternativamente $M / V L$. Este parámetro es conocido como el cociente del claro de cortante y puede entenderse como una relación de aspecto efectiva $H_{e} / L$ con $H_{e}=M / V$. La idea detrás de este parámetro es que diferentes condiciones de frontera y de carga pueden acomodarse en la fórmula de resistencia (figura 1). Es interesante notar que las fórmulas que se muestran en la tabla 1 son aplicables solamente si $M / V L \leq 1$. Tal limitación excluye los casos que corresponden a muros esbeltos y muros que tienen un momento considerable en su extremo superior $\left(M_{a}\right)$, que es una condición de carga a la que se ven sometidos ante sismo muros que son parte de un edificio de varios niveles.

En la mayoría de las fórmulas de diseño se pueden reconocer tres componentes de la resistencia a corte: 1) la resistencia a corte de la mampostería sin considerar la carga axial, 2) la contribución de la carga axial y 3) la participación del refuerzo. Muy pocas fórmulas incluyen en la resistencia el efecto de dovela del refuerzo vertical en los castillos. La primera componente, la resistencia a la tensión diagonal, es la que se ve afectada por el factor dependiente de la relación de aspecto. En la tabla 1, dicho factor está entre paréntesis en el primer término de cada ecuación, multiplicando a la resistencia de la mampostería a tensión diagonal. En todos los casos, el factor es igual a uno cuando $M / V d>1$ o $M / V L>1$, de acuerdo con el parámetro utilizado en cada ecuación. El factor expresa el incremento de la resistencia a cortante de la mampostería con la reducción de la relación de aspecto. El código basado en resistencia del comité conjunto de mampostería de Estados Unidos (MSJC SD 2002) y el código de Nueva Zelanda (NZS 2004) usan la misma variación, mientras que el código canadiense (CSA 2004) y el "Uniform Building Code" (UBC SD 1997) usan una variación un poco menos conservadora. Cada uno de los factores en la tabla 1, se presentan en la figura 2 en forma gráfica, en términos de $M / V L>1$, considerando $d=0.95 L$ para las fórmulas que utilizan $M / V d$.

Mientras que la resistencia es un parámetro básico en el diseño, es crucial entender el comportamiento no-lineal de los muros para implantar un diseño basado en desempeño, así como para desarrollar curvas de fragilidad de estructuras de mampostería. Aunque idealmente la caracterización del comportamiento no-lineal debe incluir la degradación de la rigidez y la resistencia, la primera ya ha sido 
utilizada en forma efectiva en simulaciones no lineales del comportamiento de estructuras de mampostería (Flores, 1996; Terán, 2009).

Este trabajo presenta resultados de un estudio dirigido a evaluar el efecto de la relación de aspecto en el comportamiento sísmico de la mampostería confinada con las características del sistema empleado en América Latina y algunos países europeos. Se presentan resultados de siete muros de mampostería confinada con distinta relación de aspecto, ensayados en forma cuasi-estática ante cargas cíclicas en su plano. Se examina el efecto de la relación de aspecto de los muros en la resistencia y rigidez laterales así como la degradación de la rigidez y la resistencia. El comportamiento detallado de cada espécimen puede consultarse en Cruz (2013).

\section{PROGRAMA EXPERIMENTAL}

Se ensayaron siete muros de mampostería confinada con distintas relaciones de aspecto (figura 3). Las relaciones de aspecto de los muros variaron desde $H / L=2.13$, correspondiente a muros esbeltos hasta $H / L=0.27$ relacionada con muros robustos. Los muros robustos son representativos de muros de colindancia. Todos los muros tuvieron la misma altura, $H=2.5 \mathrm{~m}$, y el mismo espesor de $12 \mathrm{~cm}$. Los muros se construyeron sobre vigas de cimentación de concreto reforzado ancladas al piso del laboratorio.

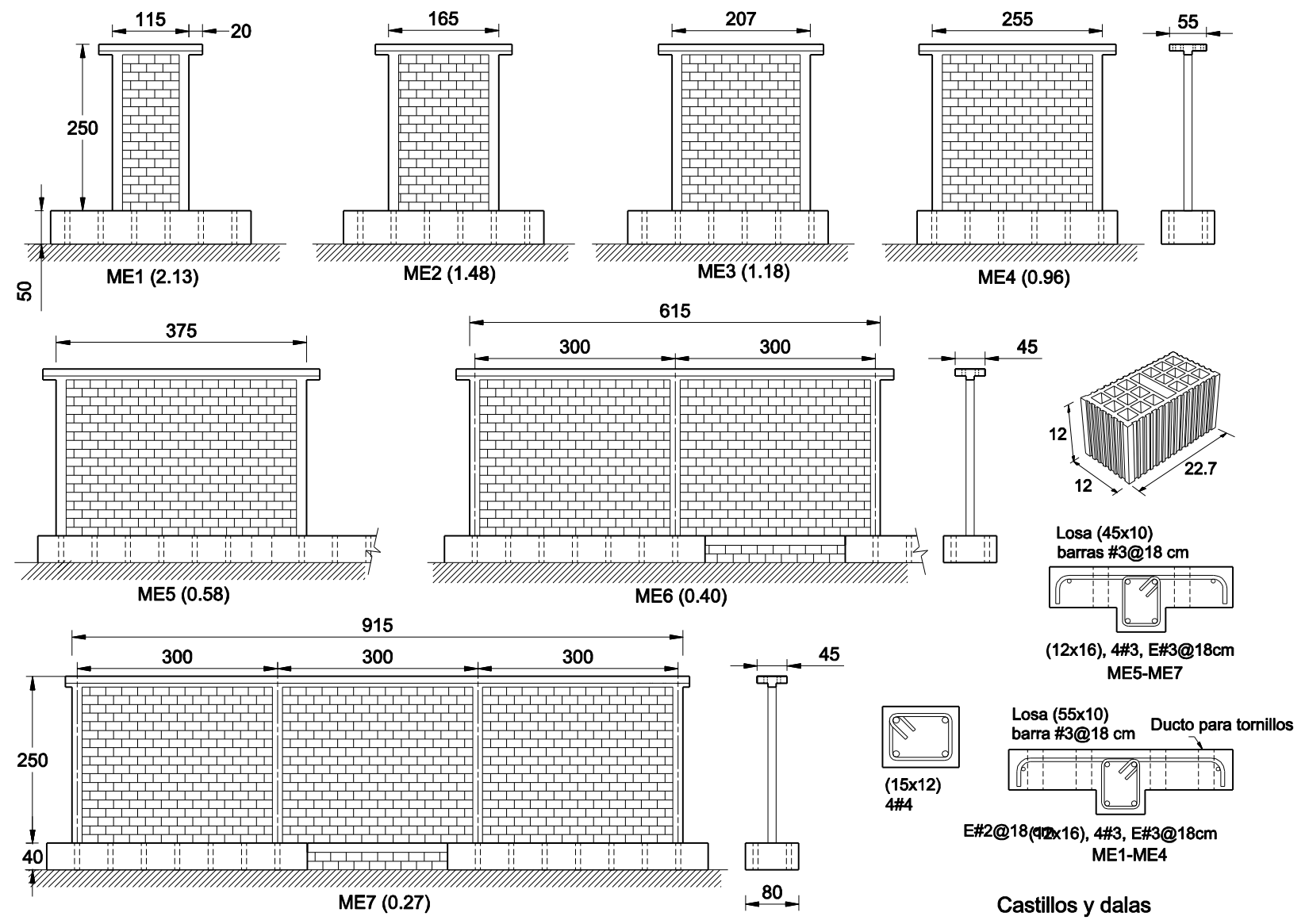

Figura 3. Características de los especímenes (dimensiones en centímetros) 


\section{Construcción de los especímenes}

Los muros fueron construidos por el mismo maestro albañil. Primeramente se construyeron los muros de mampostería; posteriormente se colaron los castillos, que en todos los casos fueron de $12 \times 15$ $\mathrm{cm}$ y finalmente se construyó la dala y losa superior de $55 \mathrm{~cm}$ de ancho para los muros ME1 a ME4 y de $45 \mathrm{~cm}$ para los muros del ME5 al ME7; en todos los muros el peralte de la losa fue de $10 \mathrm{~cm}$, formando una sección ' $\mathrm{T}$ ' con la dala rectangular de $12 \times 16 \mathrm{~cm}$.

El refuerzo longitudinal de los castillos se diseñó para promover una falla por cortante de la mampostería. Consistió de $4 \# 4$ (4 barras de $12.7 \mathrm{~mm}$ de diámetro) con un esfuerzo de fluencia nominal $f_{y}$ de $412 \mathrm{MPa}$. Se colocaron estribos del \#2 a cada $18 \mathrm{~cm}$. Se utilizaron piezas multi-perforadas de barro extruido de $12 \times 12 \times 23 \mathrm{~cm}$ (figura 3). En los últimos años, este tipo de pieza ha sido utilizado extensamente en la industria de la construcción en México, dada su relativa buena relación resistenciapeso y sus buenas características de resistencia térmica. La pieza tiene una área neta de $49 \%$, paredes interiores de $7 \mathrm{~mm}$ de espesor y paredes exteriores de $9 \mathrm{~mm}$ de espesor. Las piezas se pegaron utilizando suficiente mortero para cubrir completamente la cara perforada, dando lugar a juntas de $1 \mathrm{~cm}$ de espesor. Para mejorar la resistencia a la compresión diagonal $v_{m}$, el mortero se proporcionó para asegurar un llenado parcial de las perforaciones. En ningún caso se utilizó refuerzo interior en la mampostería. El detalle de las dimensiones de los distintos especímenes y las propiedades de los materiales se presentan en la tabla 2. También en dicha tabla, se incluyen los valores calculados y medidos de las resistencias laterales que se discuten más adelante.

Tabla 2. Geometría de los especímenes, resistencia promedio de los materiales y resistencias nominal y experimental a corte

\begin{tabular}{ccccccccccc}
\hline \multicolumn{1}{c}{ Muro } & $\begin{array}{c}L \\
(\mathrm{~m})\end{array}$ & $H / L$ & $G / E$ & $\begin{array}{c}v_{m} \\
(\mathrm{MPa})\end{array}$ & $\begin{array}{c}f_{m}^{\prime} \\
(\mathrm{MPa})\end{array}$ & $\begin{array}{c}f_{c}^{\prime} \\
(\mathrm{MPa})\end{array}$ & $\begin{array}{c}V_{n} \\
(\mathrm{kN})\end{array}$ & $\begin{array}{c}V_{\max } \\
(\mathrm{kN})\end{array}$ & $\begin{array}{c}V_{c}^{+} \\
(\mathrm{kN})\end{array}$ & $\begin{array}{c}V_{\max }^{+} \\
(\mathrm{kN})\end{array}$ \\
\hline ME1 & 1.15 & 2.13 & 0.15 & 0.305 & 5.53 & 33.4 & 41.4 & 53.8 & 45.1 & 46.0 \\
ME2 & 1.65 & 1.48 & 0.10 & 0.327 & 5.17 & 20.4 & 61.6 & 80.0 & 71.1 & 75.8 \\
ME3 & 2.07 & 1.18 & 0.11 & 0.345 & 5.57 & 18.0 & 79.4 & 103.3 & 88.4 & 98.8 \\
ME4 & 2.55 & 0.96 & 0.11 & 0.324 & 5.83 & 23.1 & 94.6 & 122.9 & 100.5 & 157.0 \\
ME5 & 3.75 & 0.58 & 0.13 & 0.457 & 8.15 & 22.7 & 191.2 & 248.5 & 251.6 & 320.8 \\
ME6 & 6.15 & 0.40 & 0.19 & 0.513 & 8.99 & 25.5 & 297.9 & 387.3 & 473.3 & 689.8 \\
ME7 & 9.15 & 0.27 & 0.20 & 0.389 & 6.54 & 29.8 & 375.4 & 488.0 & 627.8 & 835.3 \\
\hline
\end{tabular}

Nota: Las propiedades de los materiales $G$ y $v_{m}$ se obtuvieron de ensayes de muretes en compresión diagonal y $E$ y $f_{m}^{\prime}$ de ensayes de muretes en compresión; son valores promedio de 4 probetas para los muros ME1 a ME5, y 6 probetas para los muros ME6 y ME7. $f_{c}^{\prime}$ se obtuvo del ensaye de cilindros de concreto a compresión, haciendo el promedio de los resultados de 4 probetas para ME1 a ME5, 8 probetas para ME6 y 12 probetas para ME7.

\section{Arreglo del sistema de prueba y procedimiento de prueba}

El sistema de carga de los muros ME1 a ME4 se muestra en la figura 4a. La carga lateral se aplicó mediante un actuador de doble acción y la carga vertical se aplicó mediante dos actuadores verticales. La carga se aplicó en voladizo, de manera que el muro pudiera desarrollar deformaciones por flexión. Para los muros robustos, ME5 a ME7, la carga axial se aplicó por medio de tensores verticales y la carga lateral mediante la acción de dos actuadores hidráulicos. La carga horizontal se distribuyó uniformemente a lo largo de la losa, mediante una viga de acero anclada a la losa por medio de tornillos de alta resistencia, localizados simétricamente respecto al plano del muro (figura $4 b$ ). 


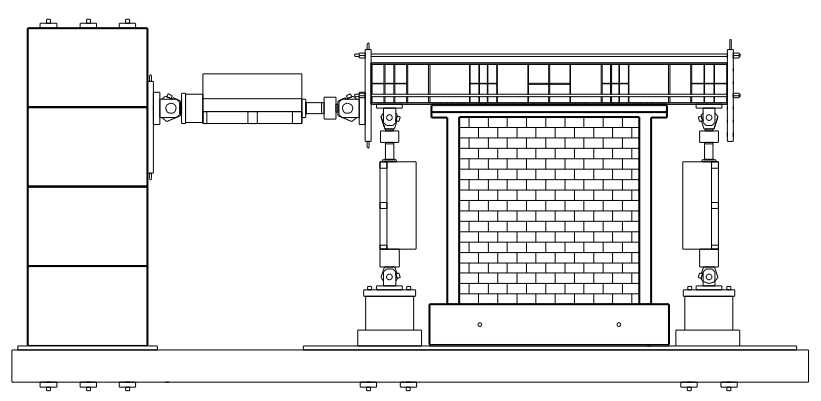

a) Arreglo experimental para muros ME1-ME4

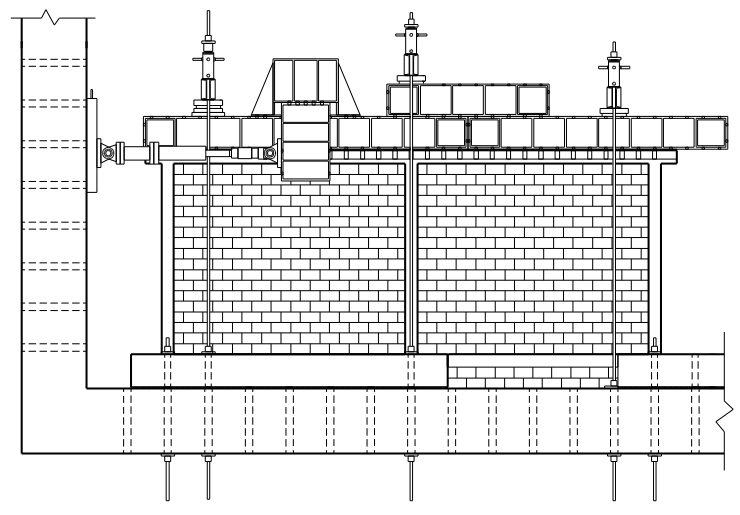

b) Arreglo experimental para muros ME5-ME7

Figura 4. Arreglo del marco de carga

Los ensayes se dividieron en dos fases: 1) la fase elástica y 2) la fase no lineal. En la fase elástica se utilizaron cuatro niveles de esfuerzo axial $\sigma=0,0.25,0.5$ y $1 \mathrm{MPa}$. Para cada nivel de esfuerzo se aplicaron ciclos de carga lateral en forma cuasi-estática usando control de fuerza de $0.25 V_{n}$ y $0.5 V_{n}$; se aplicaron dos ciclos completos para cada nivel de carga lateral. $V_{n}$ es la resistencia nominal calculada con las dimensiones de los muros y las propiedades de los materiales, de acuerdo con las Normas Técnicas Complementarias para el Diseño y Construcción de Estructuras de Mampostería del Distrito Federal (NTCM 2004). Se utilizó un factor de resistencia unitario $\left(F_{R}=1.0\right)$. Antes de iniciar la fase no lineal, el esfuerzo axial se fijó en $0.5 \mathrm{MPa}$; posteriormente, aun en control de carga se aplicó carga lateral hasta llegar al primer agrietamiento inclinado. De este punto en adelante se aplicaron ciclos con control de desplazamientos hasta alcanzar las distorsiones (desplazamiento lateral dividido por la altura del muro) de $0.002,0.004,0.006,0.008$, etc. Para cada nivel de distorsión se aplicaron dos ciclos completos hasta alcanzar la falla. La falla se estableció cuando la carga lateral se redujo en más de $20 \%$ de la resistencia medida.

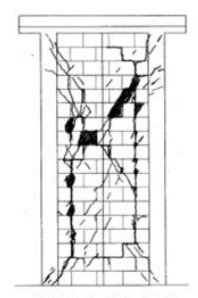

ME1 (2.13)

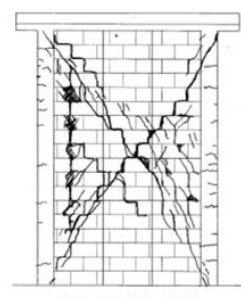

ME2 (1.48)

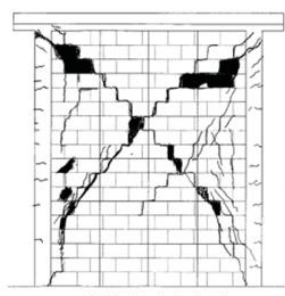

ME3 (1.18)

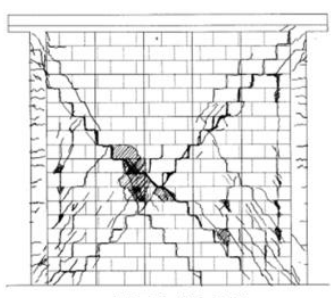

ME4 (0.96)

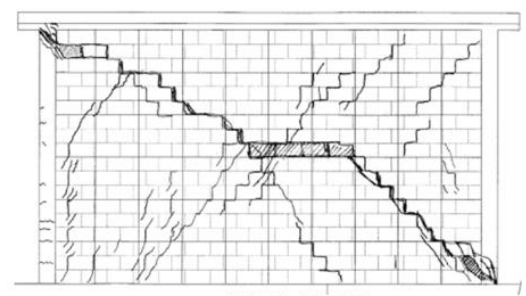

ME5 (0.58)

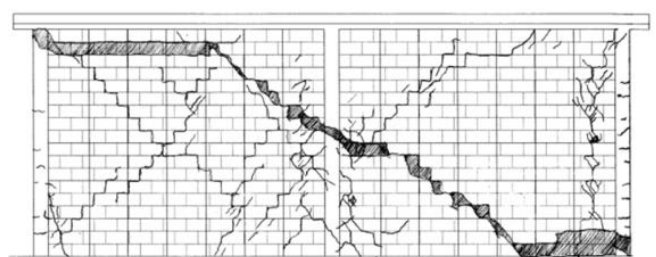

ME6 (0.40)

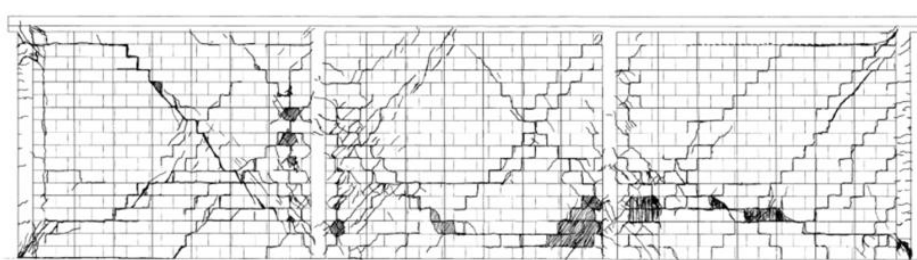

ME7 (0.27)

Figura 5. Patrones finales de agrietamiento de los muros ensayados; relación de aspecto en paréntesis 
Tabla 3. Deformaciones laterales al agrietamiento, cortante máximo y último

\begin{tabular}{cccccccc}
\hline Wall & $\begin{array}{c}L \\
(\mathrm{~m})\end{array}$ & $H / L$ & $\gamma_{c}^{+}$ & $\gamma_{m}^{+}$ & $\gamma_{u}^{+}$ & $\gamma_{m}^{+} / \gamma_{c}^{+}$ & $\gamma_{u}^{+} / \gamma_{c}^{+}$ \\
\hline ME1 & 1.15 & 2.13 & 0.0015 & 0.0040 & 0.0165 & 2.7 & 11.2 \\
ME2 & 1.65 & 1.48 & 0.0015 & 0.0039 & 0.0067 & 2.5 & 4.3 \\
ME3 & 2.07 & 1.18 & 0.0018 & 0.0025 & 0.0051 & 1.4 & 2.9 \\
ME4 & 2.55 & 0.96 & 0.0008 & 0.0038 & 0.0056 & 4.6 & 6.7 \\
ME5 & 3.75 & 0.58 & 0.0008 & 0.0035 & 0.0035 & 4.4 & 4.4 \\
ME6 & 6.15 & 0.40 & 0.0010 & 0.0032 & 0.0032 & 3.0 & 3.0 \\
ME7 & 9.15 & 0.27 & 0.0009 & 0.0019 & 0.0041 & 2.2 & 4.7 \\
\hline
\end{tabular}
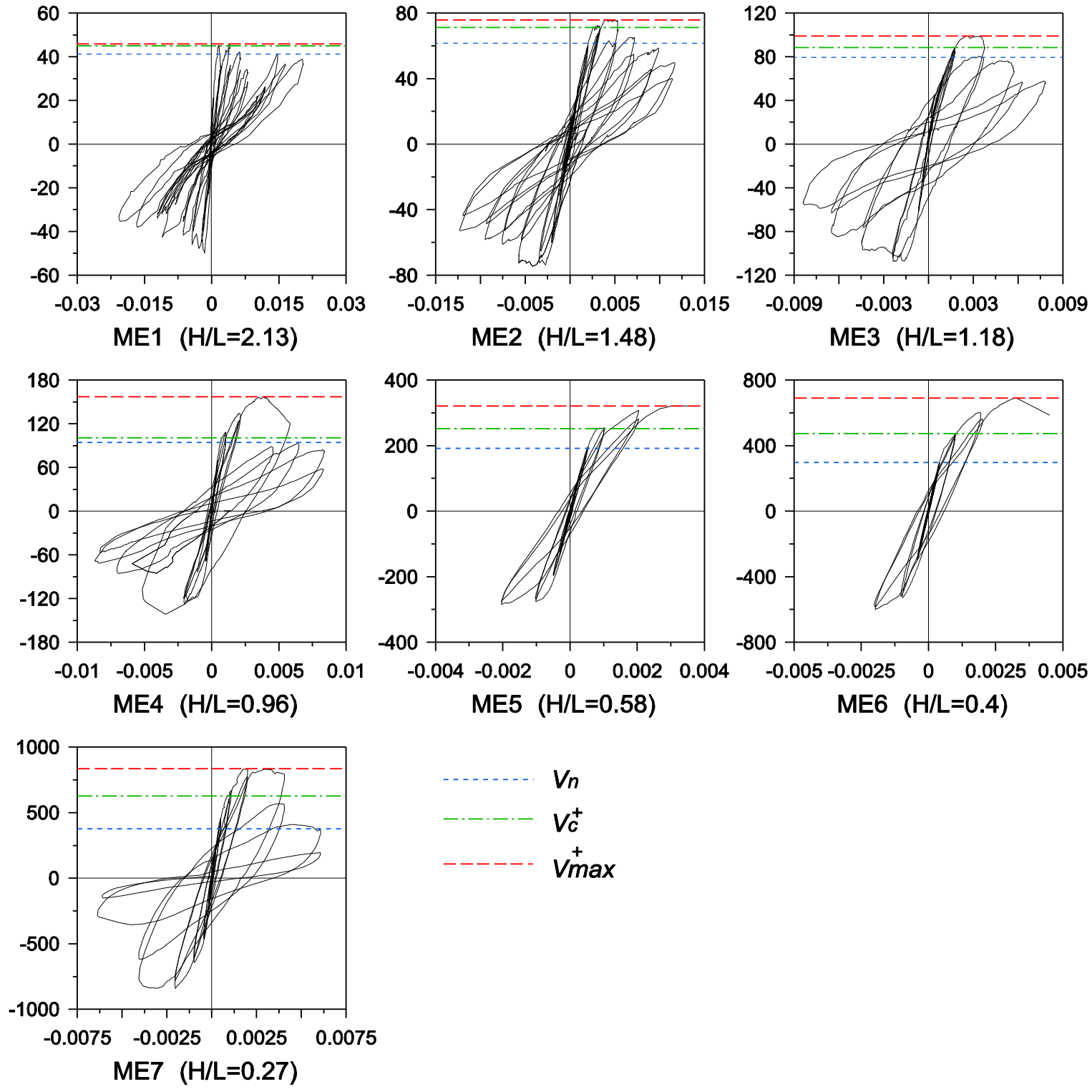

Figura 6. Curvas de histéresis, cortante $(\mathrm{kN})$ - distorsión $(\mathrm{mm} / \mathrm{mm})$ 


\section{RESULTADOS}

\section{Respuesta histerética y patrones de agrietamiento}

Los patrones de agrietamiento final y las curvas de histéresis se presentan en las figuras 5 y 6 , respectivamente. En las descripciones que siguen, se hace referencia a las envolventes de las curvas de histéresis que se presentan en la figura 11 y la degradación de las resistencias que se presentan en la figura 7. La rigidez es la pendiente en cada punto de dichas envolventes. La rigidez de ciclo que se utiliza para estudiar la degradación de la rigidez, se define más adelante. El resumen de las distorsiones al primer agrietamiento, a la resistencia y a la falla se presenta en la tabla 3.

El muro ME1 $(H / L=2.13)$ sufrió el primer agrietamiento inclinado ante una carga lateral de 45.1 $\mathrm{kN}$ y una distorsión de $0.15 \%$, alcanzando, casi inmediatamente, su resistencia $(46 \mathrm{kN})\left(V_{\max }^{+} / V_{c}^{+}=\right.$ 1.02). La rigidez lateral del muro no presentó un cambio significativo hasta dicho punto, iniciando después la degradación de la resistencia que continuó a una tasa constante con la deformación aplicada. Las envolventes positiva y negativa bien pudieran representarse con una curva bilineal. Se observan ciclos de histéresis estrechos (rama ascendente y descendente muy cerca una de la otra) que son indicativos de una baja disipación de energía. El muro desarrolló agrietamiento inclinado, como se esperaba, iniciando al centro del muro con una trayectoria cercana a $45^{\circ}$ a partir de la horizontal, con dirección al castillo, sin llegar a él. Se desarrollaron grietas verticales paralelas al castillo a una distancia aproximada de media pieza desde el borde interior del castillo. Aunque la prueba continuó hasta deformaciones del 2\%, las grietas no penetraron en los castillos. Se presentaron desprendimientos de las caras de las piezas a lo largo de las grietas inclinadas principales y a lo largo de las grietas verticales mencionadas anteriormente. Las zonas entre las grietas principales se deslizaban a lo largo de las grietas que las limitaban, sin que se desarrollaran nuevos agrietamientos al interior de dichas zonas.

El muro ME2 $(H / L=1.48)$ alcanzó su carga de agrietamiento inclinado $(71.1 \mathrm{kN})$ a una distorsión de $0.15 \%$. Fue evidente una reducción de la rigidez justo antes de alcanzar la resistencia $(75.8 \mathrm{kN})$ aun mostrando una bajo cociente $V_{\max }^{+} / V_{c}^{+}=1.07$. La degradación de la resistencia tuvo una tasa constante con la deformación, mayor que la observada en el muro ME1. Adicionalmente, los ciclos de histéresis aparecen menos estrechos, pero igualmente estables, indicando una mayor disipación de energía. Dada la menor relación de aspecto del muro, las grietas llegaron más cerca de las esquinas que en el caso del muro ME1. El final de la prueba se presentó a una distorsión del $0.67 \%$, cuando una grieta penetró el castillo en su parte superior.

El muro ME3 $(H / L=1.18)$ alcanzó la carga de agrietamiento $(88.4 \mathrm{kN})$, a un nivel de distorsión del $0.18 \%$. A partir de ese punto se aprecia un cambio gradual de la rigidez hasta llegar a la resistencia $(98.8 \mathrm{kN})$, con un aumento en el cociente $V_{\max }^{+} / V_{c}^{+}=1.12$. La degradación de la resistencia es más pronunciada en comparación con los especímenes anteriores, pero aún a tasa constante con la deformación. Los ciclos de histéresis aparecen más amplios en comparación con los muros ME1 y ME2. Se generaron las grietas inclinadas y las verticales descritas anteriormente, pero estas últimas tardaron más en desarrollarse, apareciendo inicialmente como grietas muy delgadas. En contraparte, la parte superior de los castillos fue penetrada por grietas inclinadas en etapas más tempranas. El aplastamiento de piezas produjo pedazos más grandes, tanto como una cara completa de una pieza. Se detuvo la prueba a un nivel de distorsión de $0.51 \%, 25 \%$ más pequeña que para el muro ME2.

El muro ME4 $(H / L=0.96)$ muestra un cambio significativo en el nivel de distorsión al primer agrietamiento $(0.08 \%)\left(V_{c}^{+}=100.5 \mathrm{kN}\right)$ comparado con el del muro ME3. Una segunda rama en la envolvente se puede seguir claramente desde el agrietamiento a la resistencia $(157 \mathrm{kN})$. En este muro se presentó un elevado cociente $V_{\max }^{+} / V_{c}^{+}=1.56$. La degradación de la resistencia fue más rápida y los 
ciclos de histéresis fueron menos estables en este muro que en el muro ME3, dado que se aprecia una degradación de su forma. Un modelo trilineal podría describir adecuadamente la envolvente del diagrama de histéresis. La secuencia de agrietamiento sigue un patrón muy similar al del muro ME3, sin embargo, la rotura de las piezas y los caídos mostraron una mayor fragilidad. La falla se alcanzó a una distorsión del $0.56 \%$.

El muro ME5 $(H / L=0.59)$ alcanzó su carga de agrietamiento $(251.6 \mathrm{kN})$ a un nivel de distorsión del $0.08 \%$. A partir de ese punto, la rigidez cambió en forma progresiva hasta alcanzar la resistencia a corte. Por la forma de los ciclos de histéresis, se disipó poca energía antes de la falla. Las grietas inclinadas iniciaron al centro del muro con una trayectoria hacia la dala y la viga de cimentación; posteriormente, se desarrolló un segundo conjunto de grietas, esta vez desde las esquinas, paralelas a las desarrolladas anteriormente. Se presentaron grietas verticales paralelas a uno de los castillos poco antes de la falla. La falla se presentó por deslizamiento cuando se generó una grieta horizontal a través del centro del muro y se extendió desde el centro hasta interceptar las grietas provenientes de las esquinas, causando la penetración completa de los castillos por las grietas. El número de caídos fue escaso y aconteció cerca del punto donde los castillos fueron cizallados. La distorsión a la falla fue del $0.35 \%$, que coincidió con la resistencia a cortante.

En el muro ME6 $(H / L=0.4)$ se alcanzó la carga de agrietamiento $(251.6 \mathrm{kN})$ a una distorsión de $0.1 \%$. Después de ese punto, como en el caso del muro ME5 la rigidez se degradó gradualmente hasta la resistencia alcanzando el cociente $V_{\max }^{+} / V_{c}^{+}=1.46$, cuando el muro falló en forma súbita. Se desarrolló un campo de grietas inclinadas en ambas direcciones, interrumpidas por el castillo central y, en muchas ocasiones, continuando después del mismo. Se observó una gran concentración de pequeñas grietas en la vecindad del castillo central, que aunque inclinadas, crearon un patrón vertical. En el extremo lejano del muro se desarrolló, tempranamente, una grieta vertical (siendo el lado cercano el lado donde se aplicó la carga lateral). La falla se presentó cuando se desarrollaron grietas horizontales: una desde el extremo superior cercano del muro y otra desde el extremo inferior lejano del muro, que penetraron los castillos, conectándose después con la grieta inclinada principal que atravesó el castillo central. La deformación en este punto fue de $\mathbf{0 . 3 2 \%}$. Los caídos se presentaron solo a lo largo de la grieta principal que causó la falla.

La carga de agrietamiento del muro $\operatorname{ME7}(H / L=0.27,627.8 \mathrm{kN})$ se obtuvo a una distorsión de $0.09 \%$. Como en el caso del muro ME6, la rigidez se degradó en forma gradual hasta alcanzar la resistencia. La resistencia se mantuvo constante desde una deformación de $0.16 \%$ hasta una de $0.39 \%$, a partir de la cual se observó una pronunciada caída de la resistencia. La mayor parte del diagrama de histéresis exhibe ciclos muy estrechos lo que indica que la falla fue controlada por corte. Como se explicará más adelante la disipación de energía fue baja.

En ME7 se desarrolló un campo de grietas inclinadas en ambas direcciones, con una concentración considerable de grietas en la vecindad de los castillos centrales. Llama la atención el hecho de no se hayan presentado grietas en la parte media del tablero central, dejando esta región sin daño. La falla se produjo por deslizamiento a lo largo de una grieta casi horizontal empezando y terminando en la parte inferior de los castillos extremos a los que penetró. Estas grietas se propagaron con una pendiente muy baja hasta llegar a los castillos intermedios cruzándolos. En el tablero central, la grieta descendió nuevamente para propagarse cerca de la base del muro. La deformación a la falla se registro en $0.41 \%$.

\section{DISCUSIÓN}

Las características del comportamiento de los muros ensayados en términos de los patrones de agrietamiento, resistencia, y las degradaciones de rigidez y resistencia se discuten a continuación. 


\section{Cociente entre el cortante resistente y la fuerza cortante al agrietamiento}

Como se indicó en la sección anterior, el cociente del cortante resistente y el cortante al agrietamiento se incrementó con la reducción de la relación de aspecto; el intervalo del cociente fue de 1.02 a 1.56, con una media de 1.3. Este resultado es importante ya que la mayoría de los modelos de comportamiento describen un comportamiento trilineal donde el cortante resistente es calculado multiplicando el cortante al agrietamiento por un factor que es constante para cualquier longitud de muro. De la evidencia recogida de estas pruebas, dicho factor es inapropiado para muros esbeltos.

\section{Degradación de la resistencia}

Mientras que en los muros esbeltos la resistencia es apenas mayor al cortante al primer agrietamiento diagonal, la degradación de su resistencia es más lenta que la de los muros robustos. Como se observa en la figura 7, los muros ME4 a ME1 reducen gradualmente la tasa de la degradación de su resistencia (figura 7). Este efecto se atribuye al confinamiento que proveen los castillos, el cual depende, a su vez, de la distancia entre ellos, que para muros con un solo tablero es función de la relación de aspecto. Para los muros robustos ME5 y ME6, la capacidad resistente a cargas laterales disminuyó abruptamente después de alcanzar la resistencia. El espécimen ME7 tuvo una degradación muy rápida. La degradación de la resistencia depende del mecanismo de falla que los muros desarrollaron: una combinación de deslizamiento y tensión diagonal. La razón es que a través de las grietas inclinadas se pueden transmitir fuerzas normales a la grieta, transfiriendo parte de la fuerza cortante a otras partes del muro, generando así más grietas. Una vez que se desarrolla una grieta horizontal, el mecanismo de transferencia de cortante es afectado severamente ya que muy poco cortante puede transmitirse a lo largo de la grieta horizontal. Este fenómeno conduce a una concentración de cortante en el castillo causando que éste o éstos, según el caso, fallen, produciendo la falla del muro, como se observó en todos los especímenes probados.
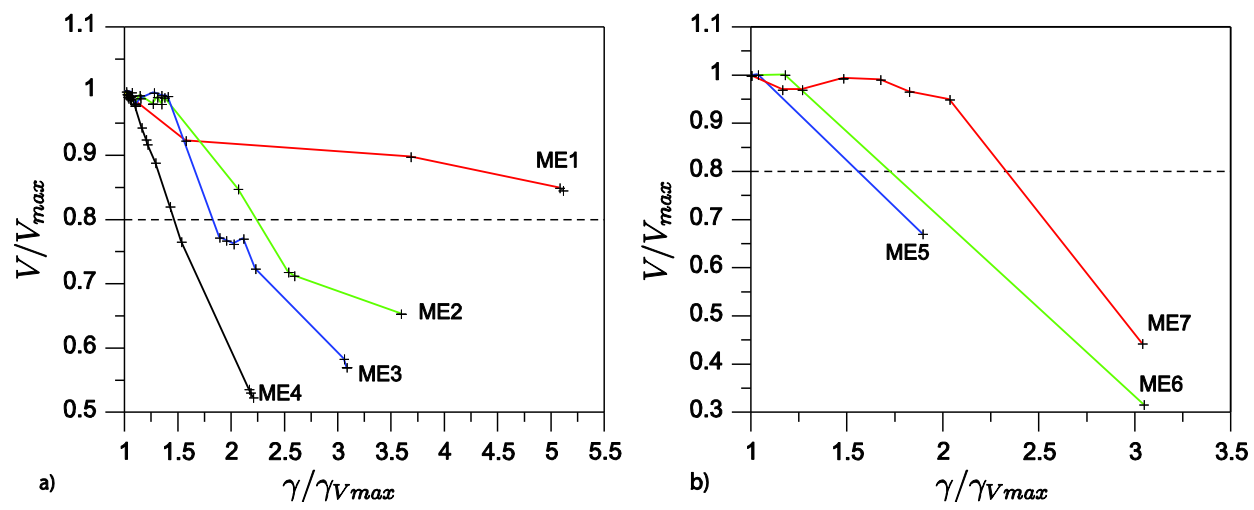

Figura 7. Degradación de resistencia, a) muros ME1 a ME4 y b) muros ME5 a ME7

\section{Disipación de energía}

Para tener una idea cuantitativa de la energía disipada, se estimó la energía disipada equivalente de acuerdo con las NTCM. Para los muros ME1 a ME4 se utilizó el valor de $\theta_{1}=0.004$ para piezas huecas. Para los muro ME4 y ME7 se uso el área del primer ciclo a dicha distorsión debido a que el segundo ciclo presentó una resistencia menor al $\mathbf{8 0 \%}$ de la máxima, esto es, el muro ya había fallado. Para los muros ME5 y ME6 se utilizó el área del último ciclo completo que en ambos casos fue para una distorsión de $\theta_{1}=0.002$ (tabla 4). La energía disipada creció de ME1 a ME3 reduciéndose ligeramente en ME4. Como era de esperarse la disipación de energía está asociada con las deformaciones por cortante y, de ahí, su relación con $H / L$. De la tabla 4 , se aprecia que el mecanismo de falla en los muros robustos, a base de una 
combinación de grietas inclinadas y horizontales, no permitió una gran disipación de energía, de acuerdo a lo descrito arriba.

Tabla 4. Energía disipada equivalente

\begin{tabular}{cccccccc}
\hline Variable & ME1 & ME2 & ME3 & ME4 & ME5 & ME6 & ME7 \\
\hline$H / L$ & 2.13 & 1.48 & 1.18 & 0.96 & 0.58 & 0.40 & 0.27 \\
$\theta_{1}$ & 0.004 & 0.004 & 0.004 & 0.004 & 0.002 & 0.002 & 0.004 \\
Energía disipada equivalente & 0.13 & 0.35 & 0.66 & 0.57 & 0.17 & 0.21 & 0.35 \\
\hline
\end{tabular}

\section{Deformaciones a la falla y ductilidad}

La distorsión alcanzada a la falla creció con la relación de aspecto (tabla 3), en el intervalo de $0.32 \%$ a $1.65 \%$. Sin embargo, no se observó una tendencia clara de la capacidad de ductilidad de los muros. La ductilidad se calculó dividiendo la distorsión a la falla entre la distorsión al agrietamiento. El valor de la ductilidad de desplazamiento varió entre 2.9 y 11.2 con una media de 5.3 (figura 8). Es interesante contrastar la disipación de energía y la ductilidad que parecen correlacionarse en forma inversa, al menos en los muros esbeltos (ME1 a ME4). En los muros esbeltos pueden alcanzarse grandes deformaciones después del agrietamiento, pero eso no implica gran disipación de energía.

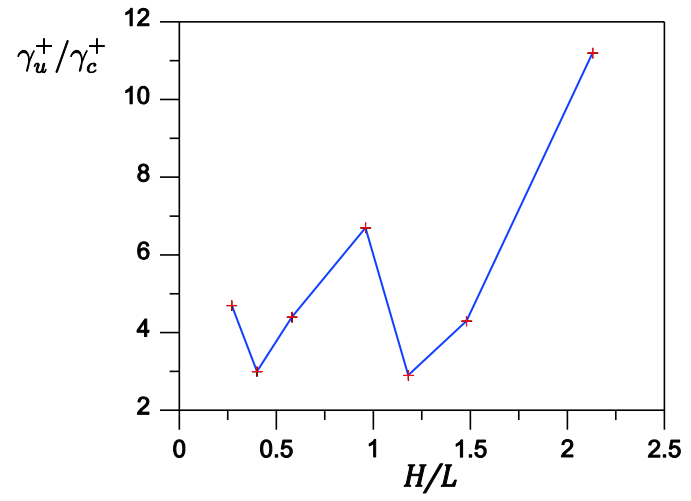

Figura 8. Ductilidad

\section{Mecanismo de falla}

Las piezas utilizadas en los especímenes se clasifican como piezas huecas de acuerdo con las NTCM. Cuando forman parte de muros sometidos a cargas cíclicas reversibles, las piezas huecas tienden a presentar un comportamiento frágil, caracterizado por aplastamiento seguido de desprendimiento de las paredes, especialmente las exteriores. Este comportamiento se observó en todas las pruebas. El desprendimiento de las paredes empezó a bajas deformaciones, del orden de $0.25 \%$ y ocurrió de forma muy frágil.

Mientras que en los muros esbeltos ME1 a ME4 el modo de falla predominante fue la tensión diagonal, en los muros robustos, ME5 a ME7, predominó la falla combinada por tensión diagonal y deslizamiento a lo largo de una o más de las juntas. La combinación de mecanismos de falla es necesaria en los muros robustos ya que debido a la relación de aspecto, las grietas diagonales de tensión que se propagan aproximadamente a 45 grados, no pueden extenderse para formar una superficie de falla. Se forman, en cambio, varias grietas inclinadas hasta que una o más grietas horizontales las interceptan para formar la superficie necesaria para la falla del muro. El muro ME7 falló por deslizamiento a lo largo de una grieta casi horizontal. En dicho muro, se distinguen en los patrones de agrietamiento registrados 
durante la prueba (figura 5), otras superficies de falla posibles con combinaciones de grietas inclinadas y horizontales, siendo la grieta horizontal una de las dos o tres posibilidades claras de falla. Es importante resaltar que los tableros de los muros ME6 y ME7 no se comportaron como el tablero de un muro aislado con la misma relación de aspecto, esto es evidente particularmente en el tablero central del muro ME7 que concentró agrietamiento en la vecindad de los castillos y la cimentación, sin que cruzara una grieta diagonal el centro.

\section{RIGIDEZ LATERAL}

\section{Rigidez de ciclo y rigidez teórica}

Para el estudio del efecto de la carga axial sobre la rigidez y su degradación, se utilizó el concepto de rigidez de ciclo. Para cada ciclo completo de carga, la rigidez de ciclo se calculó como la pendiente de la línea que une los puntos extremos del ciclo de histéresis. La distorsión correspondiente se calculó como la diferencia entre las distorsiones de dichos puntos, dividida entre dos veces la altura del muro (figura 9). La rigidez lateral teórica del muro $K_{o}$ se calculó considerando al muro en voladizo usando la ec. 1 ,

$K_{o}=\left(\frac{H^{3}}{3 E I}+\frac{\kappa H}{G A}\right)^{-1}$

donde $E$ es el módulo de elasticidad de la mampostería, $I$ es el momento de inercia de la sección calculada con la sección transformada de los castillos, $G$ es el módulo de rigidez a cortante, $A$ el área de la sección transversal y $\kappa$ el factor de cortante de la sección compuesta de mampostería y castillos de concreto aproximado como (Taveras, 2008):

$\kappa=\frac{6}{5}+\frac{6}{5} \frac{\alpha(n-1)}{2-2 \alpha}$

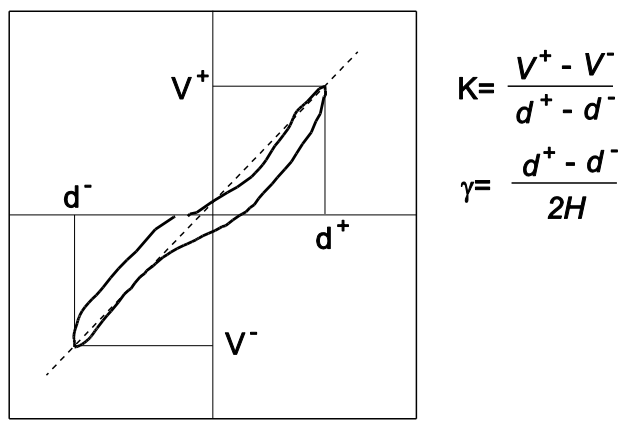

Figura 9. Rigidez de ciclo

donde $\alpha$ es el cociente de la suma de las áreas de los castillos y el área total del muro y $n$ es la relación modular, definida como el cociente entre el módulo de elasticidad del concreto y el módulo de elasticidad de la mampostería. Se puede verificar que cuando no hay castillos, $\alpha=0$ o bien $n=1$, y en ambos casos el factor de cortante es $6 / 5$, que es el factor de cortante para una sección rectangular. Un valor aproximado de la rigidez lateral teórica obtenida de los datos experimentales es $K_{40}$ que se calcula como la pendiente de la línea que va del origen al punto en la envolvente positiva con $0.4 V_{c}$. En el cálculo de $K_{o}$ se usaron los valores medios de las dimensiones y módulos de rigidez. 


\section{Efecto del esfuerzo axial y la relación de aspecto en la rigidez lateral.}

La rigidez de ciclo se calculó para cada ciclo, nivel de carga axial, y relación de aspecto, durante la fase elástica de las pruebas y se comparó con la rigidez teórica. Se hicieron gráficas con los resultados, sin que se observara ningún efecto de la carga axial en el valor de la rigidez lateral. La media de $K / K_{o}$ fue de 1.01 con una desviación estándar de 0.2. Al examinar la variación de estos datos con la relación de aspecto, tampoco se observó algún efecto. Los detalles se pueden consultar en (Cruz, 2013). Se verificó que la ec. 1 puede utilizarse para calcular la rigidez teórica en forma aproximada para el análisis utilizando los valores de $E$ y $G$ obtenidos a partir de los ensayes de pilas y muretes según lo establecen las NTCM 2004.

\section{Degradación de la rigidez}

Como se señaló la degradación de la rigidez se investigó utilizando la rigidez de ciclo. La rigidez normalizada $K / K_{40}$ y la ductilidad al agrietamiento $\mu=\gamma / \gamma_{c}$ se presentan en la figura 10. En la gráfica, $K$ es la rigidez de ciclo, $K_{40}$ es la pendiente de la recta del origen al punto de la envolvente donde $V=$ $0.4 V_{\max }^{+}, \gamma$ es la distorsión del ciclo, y $\gamma_{c}$ la distorsión al primer agrietamiento inclinado. La mayoría de los muros mostró un comportamiento de degradación de la rigidez similar que puede predecirse aproximadamente con la ec. 3

$$
\begin{array}{cc}
\frac{K}{K_{40}}=-0.15 \gamma / \gamma_{c}+1.1 & 4.5 \geq \gamma / \gamma_{c} \geq 0.7 \\
\frac{K}{K_{40}}=1.0 & \gamma / \gamma_{c}<0.7
\end{array}
$$

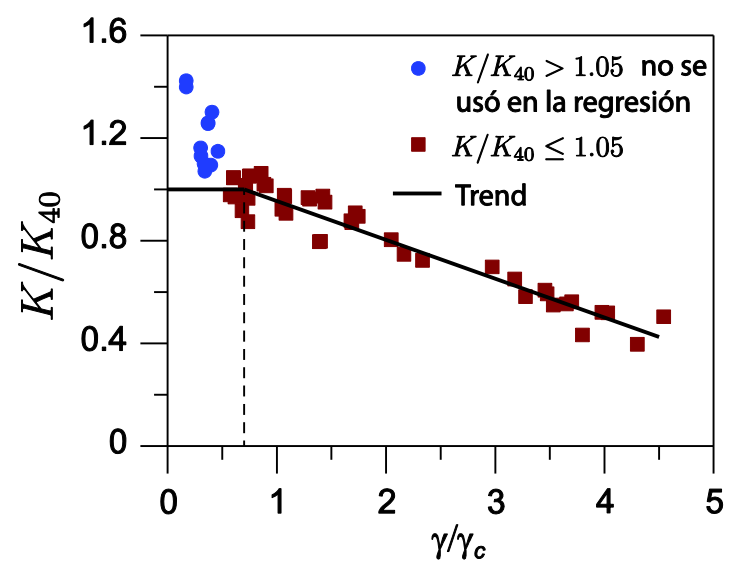

Figura 10. Degradación de rigidez de todos los muros probados

La expresión ajusta los datos con un error máximo del 11.7\% y una desviación estándar de 0.051 . Para el uso de la expresión, se observó que $K_{40}$ puede tomarse, en promedio, como $0.72 K_{0}$ donde $K_{0}$ es la rigidez lateral teórica del muro calculada con la ec. 1. Para hacer la regresión, se consideraron los puntos con $K / K_{0}<1.05$ y ductilidades menores que 4.5. El primer límite se seleccionó para evitar la zona en que la rigidez lateral cambia muy rápidamente con pequeñas variaciones de desplazamiento. La rigidez inicial se redujo rápidamente hasta llegar a $K_{40}$ que fue más estable y que se tomó como representativa de la rigidez elástica hasta una distorsión igual a $0.7 \gamma / \gamma_{c}$. El promedio de la ductilidad a la falla de los 
especímenes fue de 4.3, esto justifica el límite de ductilidad seleccionado (igual a 4.5). En el promedio mencionado, el muro ME1 no fue considerado, debido a que una capacidad de ductilidad de 11.2 fue considerada demasiado grande en comparación con el resto de los especímenes.

\section{RESISTENCIA A CORTE}

Para evaluar el efecto de la relación de aspecto en la resistencia, la resistencia experimental se normalizó con respecto a la resistencia nominal calculada con las NTCM. El esfuerzo normal fue el mismo para todos los muros. Otros autores han utilizado para la normalización una medida de la resistencia básica de la mampostería, tal como $\sqrt{f_{m}^{\prime}}$, sin embargo, la resistencia nominal es teóricamente más adecuada como se verá más adelante. En la figura 11a se muestran las envolventes de resistencias positivas y negativas normalizadas. En la figura 11b sólo se muestran las envolventes positivas.
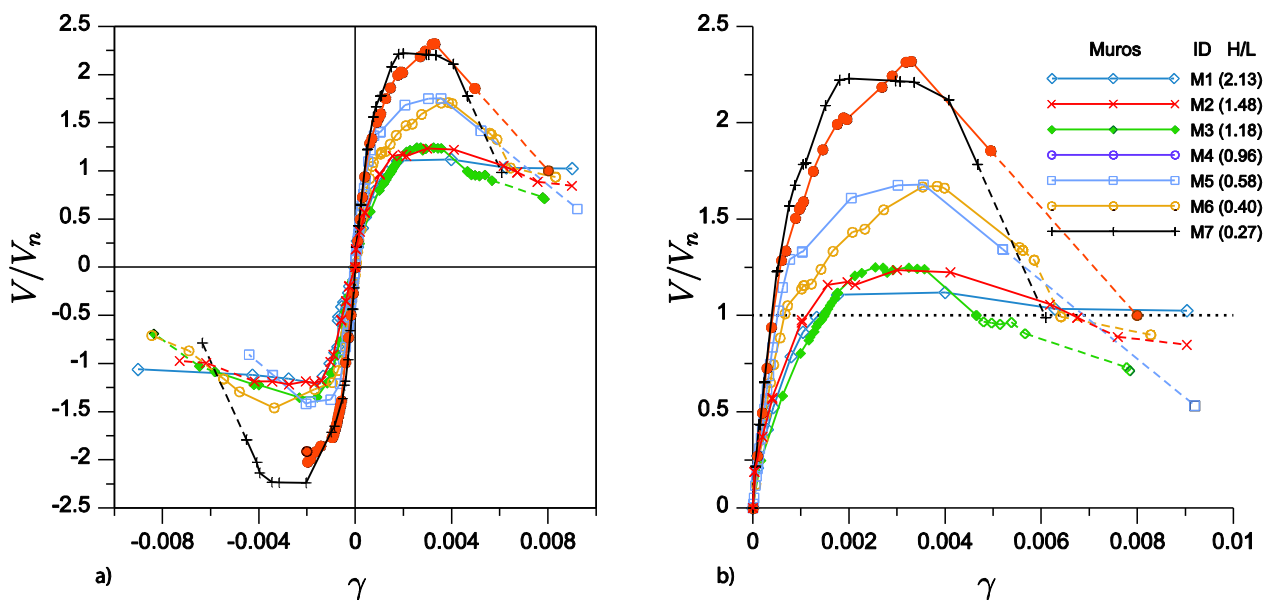

Figura 11. Envolventes de las curvas cortante - distorsión. Líneas discontinuas en la rama descendente a partir de $V=0.80 \cdot V_{\max }, V_{n}=0.5 v^{*} A+0.3 P[\mathrm{NTCM}]$ es la resistencia nominal a cortante

Dado que en la mayoría de los modelos la resistencia se basa en la resistencia al agrietamiento, es importante conocer el efecto de la relación de aspecto sobre este valor (Tomazevic et al., 1997; Meli, 1973). Comúnmente, la resistencia se calcula multiplicando el cortante al agrietamiento por un factor que varía entre 1.25 y 1.43. Con base en una extensa base de datos de muros ensayados, Riahi et al., (2009) calcularon el valor promedio de 1.3. En este estudio se vio que dicho factor no es adecuado para muros esbeltos. Adicionalmente, puede utilizarse la teoría de la elasticidad para explicar el incremento del cortante de agrietamiento observado.

\section{Explicación teórica}

La variación de la resistencia con la relación de aspecto puede atribuirse, en parte, a cambios en la deformación por flexión de los muros de distinta relación de aspecto. Esto es debido a que las fórmulas para calcular la resistencia de muros de mampostería fueron calibradas con experimentos de muros con relación de aspecto cercana a $H / L \approx 1$ sujetos a distintas condiciones de carga axial, refuerzo, etc., la geometría usada implica una relación fija de deformación debida a cortante y a momento. A medida que la relación de aspecto disminuye, esto es, a medida que los muros son más robustos, la relación de deformación por cortante y flexión cambia: la componente debida a flexión se reduce considerablemente. 
La fuerza lateral adicional observada en los muros robustos corresponde a la fuerza lateral necesaria para restituir la deformación perdida al reducirse la relación de aspecto.

Sea la deformación total requerida para producir el agrietamiento $\gamma_{c}=\gamma_{v}+\gamma_{f}$, donde $\gamma_{v}$ es la deformación por cortante y $\gamma_{f}$ la deformación por flexión. La proporción de deformación por flexión respecto al total puede escribirse como $\lambda=\gamma_{f} / \gamma_{c}$ para muros con $H / L=1$.

Para muros robustos $(H / L<1)$, este mismo cociente sería $\lambda^{\prime}=\gamma_{f}^{\prime} / \gamma_{c}^{\prime}$ donde $\lambda^{\prime}<\lambda$. Puede suponerse que $\gamma_{v}^{\prime} / \gamma_{c}^{\prime}=\gamma_{v} / \gamma_{c}$, dado que los cambios de deformación por corte con la relación de aspecto, solo dependen del área de la sección y dicha propiedad está incluida en el cálculo de la resistencia nominal. De modo que la diferencia de deformaciones entre un muro esbelto y uno robusto depende solamente de las deformaciones por flexión $\left(\lambda-\lambda^{\prime}\right)$ (figura 12). Esta cantidad es la deformación adicional que un muro robusto debe desarrollar para alcanzar el agrietamiento. Obsérvese que

$1-\frac{V_{n}^{\prime}}{V_{c}^{\prime}}=\lambda-\lambda^{\prime}$

donde $V_{n}^{\prime}$ es el valor nominal de la fuerza cortante al agrietamiento y $V_{c}^{\prime}$ es el cortante de agrietamiento que realmente se presenta. De la ec. 4 puede despejarse directamente $V_{c}^{\prime}$ para obtener:

$V_{c}^{\prime}=V_{n}^{\prime} f=V_{n}^{\prime} /\left(1-\left(\lambda-\lambda^{\prime}\right)\right)$

Siendo las deformaciones para una fuerza lateral unitaria

$\gamma_{m}=\frac{1}{12} \frac{H^{2}(4-3 \beta)}{E I} y \gamma_{v}=\frac{\kappa}{G A}$

tomando $\beta=0$ para un muro en voladizo, como los ensayados, $\eta=G / E$, donde $G$ es la rigidez a corte y $E$ el módulo de elasticidad de la mampostería, $\kappa=1.2$ el factor de forma de cortante de una sección rectangular y $w=H / L$ la relación de aspecto, se puede calcular $f$ como:

$f=\frac{(10 \eta+3)\left(10 w^{2} \eta+3\right)}{100 w^{2} \eta^{2}+60 w^{2} \eta+9}$

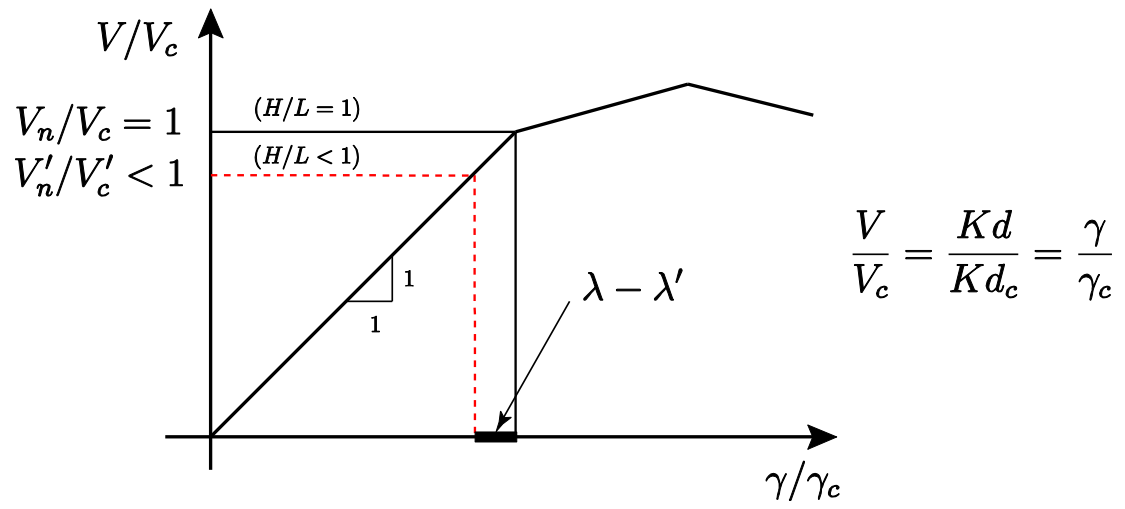

Figura 12. Deformación supuesta y real. $V_{c}$ cortante al agrietamiento, $V_{n}$ cortante al agrietamiento nominal, $d$ el desplazamiento lateral y $\gamma=d / H$. Los valores $(\cdot)^{\prime}$ corresponden a muros con $H / L<1$ 
Se puede verificar que $f(w=1)=1$, esto es la resistencia al agrietamiento de un muro cuadrado corresponde al valor nominal. El factor aumenta al reducirse $w$. Para muros con cierta restricción al giro $(\beta>0)$, y valores decrecientes de $\eta$, el factor $f$ tiende a reducirse ya que en esos casos la deformación por cortante tiende a ser más importante $\mathrm{y}$, por lo tanto, la corrección hecha por la deformación por flexión se reduce. Por ejemplo, usando $\beta=1, \eta=0.2$ y $w=0.4$, se tendrá un factor de corrección menor que para el caso en voladizo $(\beta=0)$ en un 20\%. La reducción del factor $f$ con $\beta$ es aproximadamente lineal independientemente de $w y \eta$; sin embargo, hay que tomar en cuenta que es cada vez menos probable que un muro tenga una restricción al giro importante a medida que este sea más largo $(w \rightarrow 0)$. Como se mencionó en la introducción, una forma de tomar en cuenta las condiciones de frontera es mediante el uso de una altura efectiva $H_{e}=M / V$. De la ec. $5, f=V_{c}^{\prime} / V_{n}^{\prime}$, de modo que $f$ puede entenderse como la fuerza cortante al agrietamiento experimental normalizada por el cortante nominal definido en las NTCM como:

$V_{n}=0.5 v^{*} A+0.3 P<1.5 v^{*} A$

La fórmula de las NTCM es una estimación de la fuerza que produce el agrietamiento. Ambas cantidades, $f$ calculada con los valores de $\eta=G / E$ de la tabla 2 usando la ec.7, y el valor experimental de $V_{c} / V_{n}$, se presentan en forma gráfica, en función de la relación de aspecto, en la figura 13. Se observa una buena coincidencia para muros con $H / L<1$, con errores relativos del $4.4 \%, 7.8 \%, 11.1 \%$ y $7.9 \%$ para los muros ME4 al ME7, respectivamente. La ec. 7 predice una reducción de la fuerza cortante de agrietamiento en muros con relación de aspecto $H / L>1$, en coincidencia con lo encontrado por Matsumura (1998). Sin embargo, dicha reducción no se observó en los muros ME1 a ME3. Este hecho se atribuye al confinamiento producido por la separación de los castillos que varió entre 1.15 y $2.07 \mathrm{~m}$. Para estos casos $V_{c} / V_{n}$ fue prácticamente constante.

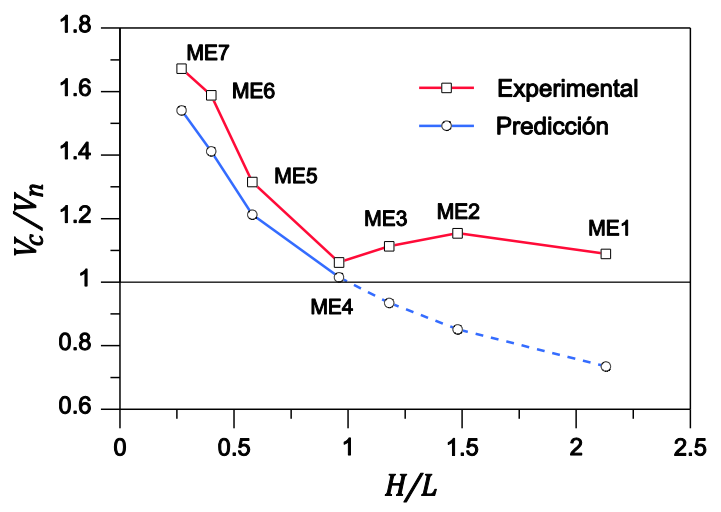

Figura 13. Fuerza cortante al agrietamiento predicha y experimental

\section{Aproximación de la fuerza cortante al agrietamiento}

La mayoría de las fórmulas para estimar la resistencia a corte que consideran la relación de aspecto de los muros, aunque no se aplican directamente a muros de mampostería confinada, corrige la componente de la resistencia debida a la mampostería (tensión diagonal) por un factor que depende de $M / V L$. En esta contribución, el factor se aplica globalmente, incluyendo a la debida al esfuerzo axial. La razón de hacerlo de esta forma es que, aunque el esfuerzo axial no afecta la rigidez lateral, sí afecta el desplazamiento lateral al agrietamiento; consecuentemente, dado que el factor $f$ es una corrección de la proporción del desplazamiento debido a cortante, este debe afectar todas las contribuciones que afecten la deformación al agrietamiento. El refuerzo horizontal, aunque no se considera en este estudio, no tiene un impacto sobre la deformación al agrietamiento (Anderson et al., 1992), por tanto esta componente no debe ser afectada por el factor de corrección. 
Por su importancia para fines de diseño es conveniente contar con una expresión simple, con adecuada precisión, del factor $f$. Para evitar el parámetro $\eta=G / E$ se supone un valor representativo. De acuerdo a varios autores (Bazán, 1980; Tomazavic, 2009) este cociente varía entre 0.1 y 0.3; se propone utilizar $\eta=0.2$. Si además se limita la relación de aspecto al intervalo entre 0.2 y 1.0 , aplicando el método de mínimos cuadrados para ajustar una recta a la ec. 7 , forzando a que $f(w=1)=1$ y utilizando una relación de aspecto efectiva, el valor de $f$ puede escribirse como:

$$
\begin{array}{ll}
f=1.55 & \frac{H_{e}}{L}<0.2 \\
f=1.69-0.69 \frac{H_{e}}{L} & 0.2 \leq \frac{H_{e}}{L} \leq 1 \\
f=1 & \frac{H_{e}}{L}>1
\end{array}
$$

De este modo, la resistencia nominal a cortante puede escribirse como:

$V_{n}=\left(0.5 v^{*} A+0.3 P\right) \cdot f<1.5 v^{*} A \cdot f$

Para facilitar la comparación entre esta propuesta y las de otros reglamentos, especialmente los incluidos en la tabla 1, se puede construir fácilmente un factor $f^{\prime}$ que afecte solamente a la resistencia básica de la mampostería, pero que conserve el valor nominal calculado con la ec. 9.

$V_{n}=\left(0.5 v^{*} A \cdot f^{\prime}+0.3 P\right)=\left(0.5 v^{*} A+0.3 P\right) \cdot f$

Usando la definición de $f$ de la ec. 9 y sustituyendo en la ec. 11 resulta en:

$f^{\prime}=\left(1+0.6 \frac{\sigma}{v^{*}}\right) \cdot\left(1.69-0.69 \frac{H_{e}}{L}\right)-0.6 \frac{\sigma}{v^{*}}$

La diferencia obvia de este factor con los de otros códigos es que el factor propuesto depende del nivel de carga axial. El factor $f^{\prime}$ se presenta en forma gráfica en la figura 14.

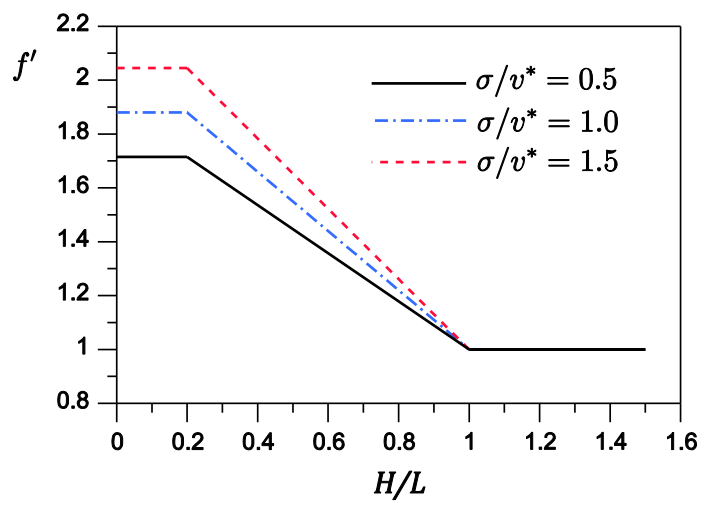

Figura 14. Factor equivalente $f^{\prime}$ aplicado solamente a la componente básica de resistencia debida a la mampostería, comparable a otros códigos

La diferencia del factor debida a esfuerzo axial se incrementa para valores decrecientes de la relación de aspecto y es también más importante para valores crecientes de $\left(\sigma / v^{*}\right)$. El efecto de la carga 
axial en la resistencia nominal a corte está limitado, de acuerdo con la ec. 8, de modo que solo es necesario considerar valores del cociente $\sigma / v^{*} \leq 3.33$.

\section{CONCLUSIONES}

De las observaciones hechas durante los ensayos y del análisis de los resultados, se pueden enumerar las siguientes conclusiones:

1. El cociente de la fuerza cortante resistente $V_{\max }$ y la fuerza cortante al agrietamiento $V_{c}$ depende de la relación de aspecto $(H / L)$, especialmente para muros con $H / L \geq 1$. Este resultado tiene un impacto en modelos de cortante-deformación que, en muchos casos, consideran el cociente $V_{\max } / V_{c}$ constante.

2. Los tableros de muros robustos con castillos intermedios no pueden considerarse como muros aislados con la misma relación de aspecto que el tablero. La importancia de este hecho es que el comportamiento de los muros no puede deducirse directamente de los tableros en forma individual, como suele suponerse en el diseño.

3. La falla de los muros con $H / L<1$ es por una combinación de tensión diagonal y deslizamiento. Las superficies de fluencia se forman de grietas horizontales e inclinadas. Este tipo de falla tiende a ser frágil.

4. La distorsión a la falla se incrementa con la relación de aspecto; sin embargo, la capacidad de deformación no depende de la relación de aspecto.

5. La rigidez lateral relativa a la teórica no se ve afectada significativamente por el nivel del esfuerzo axial ni por la relación de aspecto.

6. La degradación de la rigidez lateral de muros de mampostería confinada, para un amplio intervalo de relaciones de aspecto puede predecirse con buena precisión utilizando una relación lineal que depende de la ductilidad $\gamma / \gamma_{c}$, hasta valores de $\gamma / \gamma_{c}=4.5$. Esta información es de utilidad en el desarrollo de simulaciones numéricas.

7. La fuerza cortante al agrietamiento se incrementa a medida que la relación de aspecto decrece; este resultado es consistente con lo reportado en la literatura. A partir de un modelo de comportamiento se dedujo un factor de corrección para modificar el cálculo de la resistencia nominal a corte de las NTCM. A diferencia de lo propuesto en otros códigos, el factor propuesto se aplica también a la componente de la resistencia debida al esfuerzo axial, por considerarlo teóricamente más consistente.

\section{RECONOCIMIENTOS}

Esta investigación fue patrocinada por la Sociedad Mexicana de Ingeniería Estructural con recursos del fondo CONAFOVI-CONACYT convenio CONAFOVI-2004-C01-6. La construcción y ensayo de los especímenes ME1 a ME4 se llevó a cabo en el Instituto de Ingeniería de la Universidad Nacional Autónoma de México (UNAM) con un arreglo utilizado previamente por Miguel Torres y Mario Rodríguez para columnas de puentes, y los especímenes ME5 a ME7 en el Laboratorio de Estructuras Grandes del Centro Nacional de Prevención de Desastres (CENAPRED). El M.I. Osiel Cruz estudiante de maestría del posgrado de Ingeniería de la UNAM organizó e hizo los primeros análisis con los resultados experimentales. Los autores agradecen el apoyo brindado. 


\section{NOTACIÓN}

Los siguientes símbolos fueron utilizados en este documento:

$A \quad=$ Área transversal del muro

$A_{e} \quad=$ Área efectiva

$d \quad=$ Peralte efectivo; distancia de la fibra extrema a compresión al centroide del área de acero a tensión.

$E \quad=$ Módulo de elasticidad

$f \quad=$ Factor de corrección de la fuerza cortante al agrietamiento por la relación de aspecto

$f^{\prime} \quad=$ Factor de corrección aplicado a la resistencia básica de la mampostería

$f_{c}^{\prime} \quad=$ Resistencia especificada a compresión del concreto de los castillos

$f_{m}^{\prime} \quad=$ Resistencia especificada a compresión de la mampostería

$F_{R} \quad=$ Factor de resistencia

$f_{y} \quad=$ Esfuerzo especificado de fluencia del acero de refuerzo

$G \quad=$ Módulo de rigidez a corte de la mampostería

$H \quad$ = Altura del muro al eje de la dala superior

$H_{e} \quad=$ Altura efectiva

$K \quad=$ Rigidez lateral del muro

$K_{0} \quad=$ Rigidez lateral teórica

$L \quad=$ Longitud total del muro

$M \quad=$ Momento flexionante

$M_{a} \quad=$ Momento flexionante en el extremo superior del muro

$n \quad=$ Relación modular entre el concreto y la mampostería

$P \quad=$ Fuerza axial

$t \quad=$ Espesor del muro

$V \quad=$ Fuerza cortante actuante en el muro

$V_{c}^{+} \quad=$ Fuerza cortante de agrietamiento inclinado medida en sentido positivo

$V_{\text {max }}=$ Resistencia calculada del muro $\left(1.3 V_{n}\right)$

$V_{\max }^{+}=$Resistencia medida en sentido positivo

$V_{n} \quad=$ Resistencia nominal a corte de muros de mampostería confinada

$v_{m} \quad=$ Esfuerzo cortante resistente de la mampostería obtenido de pruebas a compresión diagonal.

$\alpha \quad=$ Cociente de área de castillos y área total del muro

$\beta=$ Restricción al giro de un muro; $\beta=0$ en voladizo $\mathrm{y} \beta=1$ totalmente restringido

$\gamma \quad=$ Deformación total

$\gamma_{c}=$ Deformación al primer agrietamiento por tensión diagonal

$\gamma_{f} \quad=$ Deformación por flexión

$\gamma_{v} \quad=$ Deformación por cortante

$\gamma_{V \max }=$ Deformación cuando se alcanza la resistencia

$\eta \quad=G / E$

$\kappa \quad=$ Factor de cortante de una sección

$\lambda=$ Cociente del desplazamiento a flexión y el desplazamiento total con $H / L=1$

$\lambda^{\prime} \quad=$ Cociente del desplazamiento a flexión y el desplazamiento total con $H / L \leq 1$

$\mu \quad=$ Ductilidad medida al agrietamiento $\gamma / \gamma_{c}$

$\theta_{1} \quad=$ Distorsión para el cálculo de la disipación de energía equivalente del apéndice A de las NTCM

$\sigma \quad=$ Esfuerzo axial sobre el muro 


\section{REFERENCIAS}

Aguilar, G., Meli, R., Díaz, R., y Vásquez del Mercado, A. R. (1996) "Influence of horizontal reinforcement on the behavior of confined masonry walls"11th World Conf. Earthquake Engineering; Acapulco, México.

Alcocer, S., y Meli, R. (1995) "Test program on the seismic behavior of confined masonry walls", The Masonry Soc. J. (Boulder), Vol. 12,No. 2pp 68-76.

Alvarez, J. J. (1996) "Some topics of the seismic behavior of confined masonry structures". Eleventh World Conference on Earthquake Engineering, paper No 180; Elsevier Science Ltd.

Anderson, D. L., y Priestley, M. J. (1992) "In plane strength of masonry walls". Proc. 6th Canadian Masonry Symp, Saskatchewan, Sask., Canada, pp. 223-234.

Bazán, E., (1980), "Muros de mampostería ante cargas laterales”, (tesis doctoral), Posgrado de Ingeniería, UNAM, México D. F.

Chen, S. J., P. A., Hidalgo, R. L., Mayes, R.W. Clough y H. D., McNiven, (1978) "Cyclic loading tests of masonry piers, Volume 2 - height to width ratio of 1", Report No. UCB/EERC-78/28.

Cruz S., O., (2013) "Ensayes de 7 muros de mampostería confinada con distinta longitud", Editorial académica española, ISBN: 978-3-659-07627-5.

CSA. (2004), "S304.1-04 Design of masonry structures", Canadian Standard Association; Mississauga, Ontario, Canada.

Davis, C. L. (2008) "Evaluation of design provisions for in-plane shear in masonry walls". Master of Science Thesis, Washington State University, Civil Engineering.

Flores, L. E., y Alcocer, S. M. (1996) “Calculated response of confined masonry structures", 11th World Conf. on Earthquake Engineering, Acapulco, México, paper No. 1830.

Hidalgo, P.A. R. L., Mayes, H. D., McNiven y R.W. Clough, (1978) "Cyclic loading tests of masonry piers, Volume 1 - height to width ratio of 2", Report No. UCB/EERC-78/27.

Hidalgo, P.A. R. L., Mayes, H. D., McNiven y R.W. Clough, (1979) "Cyclic loading tests of masonry piers, Volume 3 - height to width ratio of 0.5", Report No. UCB/EERC-79/12.

Matsumura, A. (1998) "Shear Strength of reinforced masonry walls". Proceedings of Ninth World Conference on Earthquake Engineering Vol. VI, pp. 121-126; Tokyo-Kyoto, Japan.

Meli, R. (1973) "Behaviour of masonry walls under lateral loads", Fifth World Conference on Earthquake Engineering; Rome.

MSJC SD (2002) “TMS 402-08/ACI, 530-08/ASCE 6-08, Building Code Requirements and Specification for Masonry Structures". Masonry Standards Joint Committee.

NTCM (2004) "Normas Técnicas Complementarias para el Diseño y Construcción de Estructuras de Mampostería". Gobierno del Distrito Federal, México.

NZS (2004) "New Zealand Standard 4230:2004, Design of Reinforced Concrete Masonry Structures", Standards Association of New Zealand; Wellington.

Riahi, Z., Elwood, K. J., y Alcocer, S. M. (2009), "Backbone model for confined masonry walls for performance-based seismic design”, Journal of Structural Engineering; Vol. 135, pp 644-654.

Taveras, M. A. (2008) "Revisión de las recomendaciones para modelar y analizar estructuras de mampostería confinada ante carga lateral”, (tesis de maestría), Posgrado de Ingeniería, UNAM. 
Terán-Gilmore, A., Zuñiga-Cuevas, O., y Ruiz-García, J. (2009) "Displacement-based seismic assessment of low-height confined masonry buildings". Earthquake Spectra; Vol. 25pp. 439-464

Tomazevic, M., y Klemenc, I. (1997) "Seismic behaviour of confined masonry walls". Earthquake Engineering and Structural Dynamics, Vol. 26 No. 10, pp. 1059-1071.

Tomazevic, M., (2009) "Shear resistance of masonry walls and Eurocode 6: shear versus tensile strength of masonry", Materials and Structures, Vol. 42, pp. 889-907.

UBC SD (1997) "Uniform Building Code (Vol. 2)"; International Conference of Building Officials; Whittier, California.

Voon, K. C., e Ingham, J. M. (2006) "Experimental in-plane shear strength investigation of reinforced concrete masonry walls". Journal of Structural Engineering, Vol. 132 No. 3, pp. 400-408.

Yoshimura, K., Kikuchi, K., Okamoto, Z., y Sanchez, T. (1996) "Effect of vertical and horizontal wall reinforcement on seismic behavior of confined masonry walls". 11th World Conf. Earthquake Engineering; paper No 191; Acapulco, México. 